\title{
The large-scale distribution and physical properties of massive star forming regions in the Milky Way
}

\author{
Jarken Esimbek $^{1}$, Zhou Jianjun ${ }^{1}$ and Zheng Xingwu ${ }^{2}$ \\ ${ }^{1}$ National Astronomical Observatories/Urumqi Observatory, CAS, Urumqi 830011, China \\ email: Jarken@ms.xjb.ac.cn \\ ${ }^{2}$ Department of Astronomy, Nanjing University, Nanjing 210093, China, \\ email: xwzheng@nju.edu.cn
}

\begin{abstract}
We are conducting a large survey with the Urumqi 25 m radio telescope to study the distribution and physical properties of massive star forming regions in the Milky Way. We will accomplish this by observing the $\mathrm{H}_{110 \alpha}$ hydrogen recombination line at $4.874 \mathrm{GHz}$ and the $\mathrm{H}_{2} \mathrm{CO}$ absorption line at $4830 \mathrm{GHz}$. These lines are associated with compact HII regions and dense molecular clouds. As a test of the $6 \mathrm{~cm}$ spectral line receiving system, we observed the two spectral lines toward massive star forming regions. We plan to study the large scale distribution and physical properties of massive star forming regions in the Milky way.
\end{abstract}

Keywords. ISM: HII regions,Galaxy: kinematics and dynamics,Galaxy: structure

\section{The spectral system and observational tests}

Formadehyde $\left(\mathrm{H}_{2} \mathrm{CO}\right)$ was one of the first poly-atomic molecules to be detected in interstellar space and has since been found to be a common constituent of interstellar molecular clouds. $\mathrm{H}_{2} \mathrm{CO}$ is associated with $\mathrm{HII}$ regions and is therefore a good tracer and probe of regions of star formation. HII regions can be expected to provide crucial evidence for any spiral structure in own Galaxy. This was recognized in the bold attempt by Georgelin \& Georgelin (1976) to derive a grand spiral pattern for our Galaxy from studies of HII regions. Valuable additions to the northern hemisphere data were made by Downes et al. (1980) and to the southern hemisphere data were made by Caswell \& Haynes(1987).

We will measure the line velocities, and hence derive kinematic distances. The kinematic distance ambiguity may partly be resolved by observing the two lines simultaneously in the same band. From the survey we aim to establish the Galactic distribution of massive star forming regions. The study of global star formation will provide important information for the environment of young stars and clusters. However, global star formation still remains poorly understood.

The 25-meter radio telescope of Urumqi Astronomical Station of NAOC, is located at Nanshan Mountain near the sourth suburb of Urumqi city, Xinjiang province. It is situated $2080 \mathrm{~m}$ above sea level, at a very dry site for short centimeter observations. The Urumqi Observatory $25 \mathrm{~m}$ radio telescope has a 4096-channel digital correlator as the back end. The correlator, used with a $80 \mathrm{MHz}$ bandwidth, can simultaneously observe the $\mathrm{H}_{110 \alpha}$ hydrogen recombination line at $4.874157 \mathrm{GHz}$ and the formaldehye $\left(\mathrm{H}_{2} \mathrm{CO}\right)$ absorption line at $4.82966 \mathrm{GHz}$. In the $\mathrm{C}$ band, the $25 \mathrm{~m}$ telescope has a spatial resolution of about $10^{\prime}$, and a system temperature of about $22 \mathrm{~K}$. This is well suited to observing the two spectral lines simultaneously. We are using this system to survey the large-scale distribution and physical properties of massive star forming regions in the Milky Way. 


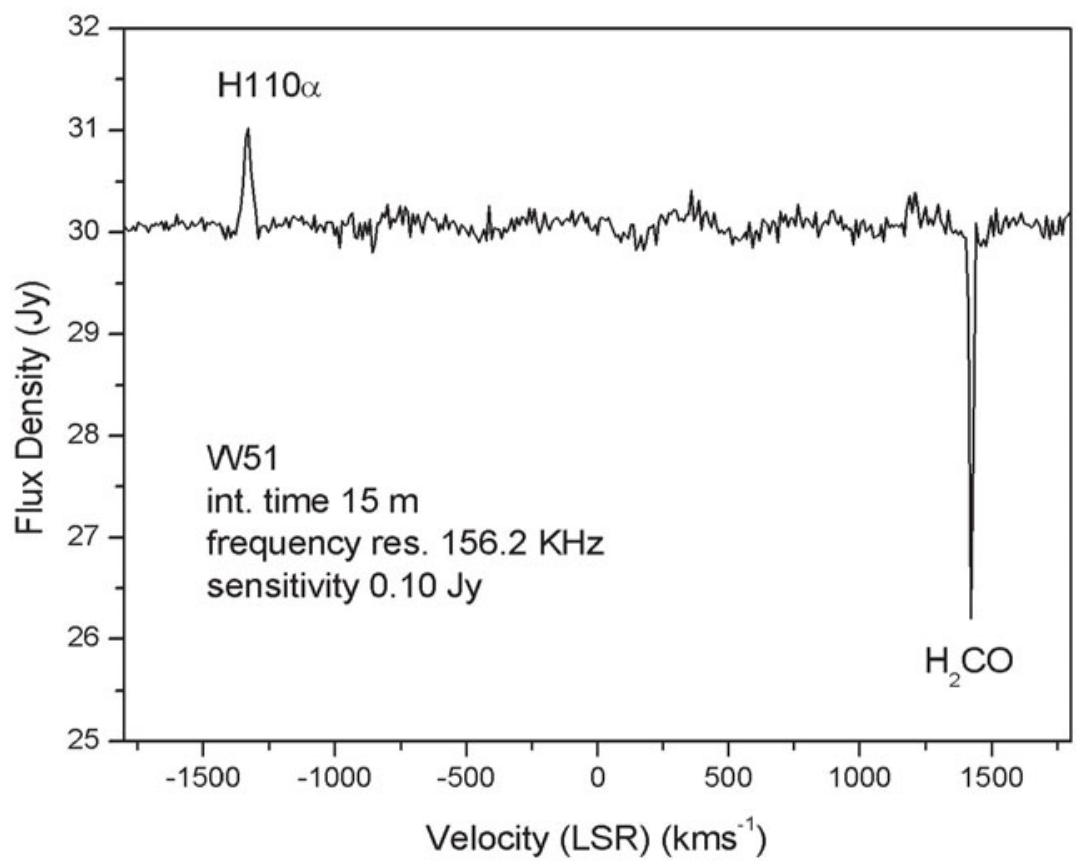

Figure 1. As a testing of $6 \mathrm{~cm}$ spectral line receiving system, we observed the two spectral lines toward W51 massive star forming regions, integration times is $15 \mathrm{~m}$, the system temperature was about $22 \mathrm{~K}$.

As a test of the $6 \mathrm{~cm}$ spectral line receiving system, we observed the two spectral lines toward massive star forming regions. An example is shown in Figure 1.

\section{Acknowledgements}

This work was financially supported by funds from the National Natural Science Foundation of China (Grant Nos. 10203003 and 10073004), and supported by the Program of the Light in China's Western Region (LCRW) under Grant No.RCPY200605.

\section{References}

Caswell, J. L. \& Haynes, R. F 1987, A\& A 171, 261C

Downes, D., Wilson, T. L., Bieging, J., \& Wink, J. 1980, A\&AS 40, 379D

Georgelin, Y. M. \& Georgelin, Y. P. 1976, A\& A 49, 57G 